\title{
Decomposition of the Total Momentum in a Linear Dielectric into Field and Matter Components
}

\author{
Michael E. Crenshaw* \\ US Army Aviation and Missile Research, Development, \\ and Engineering Center, Redstone Arsenal, AL 35898, USA
}

(Dated: August 27, 2018)

\begin{abstract}
The long-standing resolution of the Abraham-Minkowski electromagnetic momentum controversy is predicated on a decomposition of the total momentum of a closed continuum electrodynamic system into separate field and matter components. Using a microscopic model of a simple linear dielectric, we derive Lagrangian equations of motion for the electric dipoles and show that the dielectric can be treated as a collection of stationary simple harmonic oscillators that are driven by the electric field and produce a polarization field in response. The macroscopic energy and momentum are defined in terms of the electric, magnetic, and polarization fields that travel through the dielectric together as a pulse of electromagnetic radiation. We conclude that both the macroscopic total energy and the macroscopic total momentum are entirely electromagnetic in nature for a simple linear dielectric in the absence of significant reflections.
\end{abstract}

\section{INTRODUCTION}

In a dielectric environment, the momentum of the electromagnetic field becomes a contested issue. The matter was first raised over a century ago when Minkowski [1], in 1908, and Abraham [2], in 1909, produced conflicting expressions for the energy-momentum tensor of an electromagnetic field in a linear optical medium. While a number of experiments seemed to validate the Abraham momentum density

$$
\mathbf{g}_{A}=\frac{1}{c}(\mathbf{E} \times \mathbf{H})
$$

other experiments suggested that the Minkowski momentum density

$$
\mathbf{g}_{M}=\frac{1}{c}(\mathbf{D} \times \mathbf{B})
$$

provides the correct description of the momentum of light in a linear medium [3]. The inability to decide between the two energy-momentum tensors and their underlying momentum densities became known as the AbrahamMinkowski controversy. The resolution of the AbrahamMinkowski dilemma was provided by Penfield and Haus [4], based on earlier work by Møller [5], who showed that the issue is undecidable because neither the Abraham momentum nor the Minkowski momentum is the total momentum. Instead, each corresponds to an open description of the system in which the momentum is allocated differently between the field and the matter $3-8]$.

\footnotetext{
*Electronic address: michael.e.crenshaw4.civ@mail.mil NOTICE: this is the author's version of a work that was accepted for publication in Annals of Physics. Changes resulting from the publishing process, such as peer review, editing, corrections, structural formatting, and other quality control mechanisms may not be reflected in this document. Changes may have been made to this work since it was submitted for publication. A definitive version was subsequently published in Annals of Physics, 338, 97 (2013).
}

Following the work of Penfield and Haus [4], Gordon [9], in 1973, assumed the Abraham form for the field momentum subsystem and derived the material contribution to the momentum in a dilute vapor by integrating the Lorentz force on each dipole. Gordon derived the total momentum density

$$
\mathbf{g}_{G}=\frac{1}{c}(n \mathbf{E} \times \mathbf{B})
$$

for a vapor that is sufficiently dilute that reflections can be neglected. Three years later, Peierls [10] followed a similar procedure to obtain a total momentum density

$$
\mathbf{g}_{P}=\frac{n^{2}+1}{2 c}(\mathbf{E} \times \mathbf{B})
$$

that is the arithmetic mean of the Abraham and Minkowski forms. The Gordon and Peierls momentum densities are equivalent to lowest order in $\delta n=n-1$. The literature on the subject is extensive and the reader is referred to reviews of the prior work by Pfeifer, et al. [8], Barnett and Loudon [11], Milonni and Boyd 12], and Kemp [13] and the resource letter by Griffiths [14].

At this point in time, there is a strong consensus in the scientific literature that only the total energy and the total momentum have physical meaning. Then any decomposition of the total momentum into a field component and a matter component is valid as long as their sum is the total momentum [8], but the separate momentum components are not physical. Nevertheless, current practice is to acknowledge that consensus and then proceed to assume some form of field momentum, usually Abraham, Minkowski, or both, and then add some momentum for the matter, usually in the form of the movement of massive particles of the medium that are forced into motion by being either pulled or pushed by the Lorentz force. The recent experiments of She, Yu, and Feng [15], for example, are analyzed in terms of an inward push force as light exits a nanometer silica filament. In contrast, earlier experiments by Ashkin and Dziedzic [16] indicate an 
outward pull force on the surface as light enters a liquid. Pfeifer, Nieminen, Heckenberg, and Rubinsztein-Dunlop [8], and also Ramos, Rubilar, and Obukhov 17], start with the Abraham tensor for the electromagnetic field and add a dust tensor for the material and claim that the result is the total energy-momentum tensor. By definition, the total energy-momentum tensor can also be derived by starting with the Minkowski tensor and adding a different material tensor. Barnett [18] identifies the Abraham momentum as the kinematic momentum of the block of dielectric, as a whole, and the Minkowski momentum as the canonical momentum that is associated with the movement of massive particles embedded in the dielectric. Barnett goes on to say that the total momentum is both the Abraham momentum with a corresponding partial momentum and the Minkowski momentum with a different partial momentum, which is again true by definition of total momentum. Mansuripur [19] treats the kinematic momentum of partially reflecting matter, pushed by a radiation pressure associated with the Fresnel reflection, together with the forward traveling light momentum. Kemp [20] formalizes a subsystem approach for the field and matter components of momentum.

The most rigorous approach to light propagation in a material is to use a microscopic model in which the microscopic electromagnetic field interacts with individual electric dipoles in the vacuum. If the distance between dipoles in the material is much smaller than the wavelength, then the theory can be considerably simplified by applying an averaging scheme to a volume containing many atoms per cubic radiation wavelength. Gordon [9], for example, spatially averages the Lorentz force on a single electric dipole to create a macroscopic Lorentz force density. Integrating the Lorentz force density, Gordon obtains a definite formula

$$
\mathbf{g}_{\text {mat }}=\frac{n-1}{c}(\mathbf{E} \times \mathbf{B})
$$

for the material component of momentum density in the dielectric. Gordon assumes the Abraham form for the momentum density of the field, then the field plus material momentum is the total momentum

$$
\mathbf{G}_{t o t a l}=\int_{\sigma} \frac{n}{c}(\mathbf{E} \times \mathbf{B}),
$$

where integration of the momentum density is performed over a volume $V$ that includes all fields present that is extended to all space $\sigma$. Saldanha 7] and Bradshaw, Shi, Boyd, and Milonni and Boyd 21], among others, have revisited Gordon's derivation of the total momentum as the sum of the Abraham momentum for the field and a material momentum arising from the Lorentz force on dipoles and derived the Minkowski momentum, which is not conserved, for the total momentum. In recent work 22], global conservation principles were applied to a closed system consisting of a quasimonochromatic pulse of electromagnetic radiation normally incident on a stationary simple dielectric with refractive index $n$ through a gradient-index antireflection coating. Conservation of the total momentum in a thermodynamically closed system with complete equations of motion [6] is decisive. Then, despite problems with the original derivation, Eq. (6) is the proven total momentum for the light pulse plus stationary dielectric closed system [22, 23].

In this article, we investigate the interaction of the electromagnetic field with a simple linear dielectric that is modeled microscopically as a collection of individual electric dipoles composed of a positively charged particle and a negatively charged particle. The work of Penfield and Haus [4] and of Gordon [9] is reproduced in part, but we go on to show that the dielectric can be treated in the usual and familiar way as a collection of stationary simple harmonic oscillators driven by the electric field. Because the contribution to the total momentum occurs through the resulting polarization field, the total momentum can be regarded as electromagnetic in character. We begin, in Section II, with an analysis of the macroscopic picture of the total momentum as being composed of arbitrary field and matter components of momentum. At the level of continuum electrodynamics, we take the material momentum to be the difference of the total momentum, which is conserved, and a field momentum that is to be determined. The temporal derivative of the material momentum density is the macroscopic force density that can be expressed in terms of the fields and a macroscopic material property. Assuming that a decomposition of the total momentum into field and material components exists, we find that each choice of the field momentum results in a specific relation between the refractive index and the macroscopic material property. Then, the decomposition of the total momentum into field and material components is not arbitrary or degenerate, but unique and definite. In Section III, we summarize Gordon's derivation of the macroscopic Lorentz force from the microscopic Lorentz force. We discuss the problem that occur when we use the macroscopic Lorentz force, along with similar results by Peierls [10], Saldanha [7], and Bradshaw, Shi, Boyd, and Milonni 21], to obtain the field momentum. Finally, in Section IV, we adopt a microscopic model of the material as being composed of discrete dipoles formed from individual pairs of positive and negative charges. Writing the microscopic Lagrangian in a center-of-mass coordinate system, we derive Lagrange equations of motion using both the center-of-mass coordinates and the relative coordinates. The microscopic Lorentz force used by Gordon [9], Penfield and Haus [4], and other authors can be extracted from the Lagrange equations for the center-of-mass coordinates. However, macroscopic movement of massive particles is not allowed in a solid block of dielectric. Setting the velocity of the center-of-mass of the dipoles to zero in the Lagrange equations for the relative coordinates, we find the elementary and expected result that the dielectric can be treated as a collection of stationary simple harmonic oscillators driven by the electric field 24]. The polarization field that is generated by the oscillators contributes to both the total energy and 
the total momentum through the refractive index $n$. We conclude that, in the absence of reflections, the total energy and the total momentum are electromagnetic in nature and do not separate into field and material motion components.

\section{FIELD AND MATTER COMPONENTS OF THE TOTAL MOMENTUM}

A physical theory is an abstract mathematical model of some limited aspect of the real world [25], in this case, light traveling at speed $c / n$ in a simple linear dielectric. Our model dielectric consists of a rectangular prism of space in which light travels at speed $c / n$, where $n$ is a real constant. No such idealization of a dielectric exists in the real world. Instead, the usual conditions for results of the model to correspond to results in the real world apply: a simple dielectric is linear, isotropic, homogeneous, transparent, and dispersion-negligible in a regime in which electrostrictive effects and magnetostrictive effects can be neglected [26]. We assume that a quasimonochromatic optical pulse impinges on the dielectric from the vacuum at normal incidence in the plane-wave limit and that the dielectric is covered with a thin gradient-index coating in order to make reflections and surface radiation pressure negligible.

The Abraham momentum is a common, but not exclusive, choice for the momentum of the field in a dielectric [8, 9, 12, 19]. Rather than make this an ansatz, we start with a more general form of the field momentum in a dielectric

$$
\mathbf{G}_{f i e l d}=\int_{\sigma} \frac{\zeta(n)}{c}(\mathbf{E} \times \mathbf{B}) d v
$$

with $\zeta(n)$ an unspecified function of $n$, that covers most of the proposed forms of electromagnetic momentum, (e.g., the Abraham, Minkowski, and Peirels momentums). We note that $\zeta$ is specifically not a function of the electric and magnetic fields. In addition, $\zeta$ is not arbitrary, but a definite function of macroscopic property constants that is to be determined. The momentum of the material $\mathbf{G}_{m a t}$ is the difference of the total momentum, Eq. (6) and field momentum, Eq. (7). Then

$$
\mathbf{G}_{m a t}=\mathbf{G}_{t o t a l}-\mathbf{G}_{f i e l d}=\int_{\sigma} \frac{n-\zeta(n)}{c}(\mathbf{E} \times \mathbf{B}) d v .
$$

The material momentum density

$$
\mathbf{g}_{m a t}=\frac{n-\zeta(n)}{c}(\mathbf{E} \times \mathbf{B})
$$

leads directly to a macroscopic force density

$$
\mathbf{f}_{m a t}=\frac{\partial \mathbf{g}_{m a t}}{\partial t}=(n-\zeta(n))\left(\frac{\partial \mathbf{E}}{\partial(c t)} \times \mathbf{B}+\mathbf{E} \times \frac{\partial \mathbf{B}}{\partial(c t)}\right) .
$$

We define a macroscopic material property

$$
\xi_{e}(n)=n-\zeta(n)
$$

that relates the electric and magnetic fields to a macroscopic force density

$$
\mathbf{f}_{m a t}=\xi_{e}\left(\frac{\partial \mathbf{E}}{\partial(c t)} \times \mathbf{B}+\mathbf{E} \times \frac{\partial \mathbf{B}}{\partial(c t)}\right)
$$

on the spatially distributed particles of matter. Then the decomposition of the total momentum into field and matter components,

$$
\begin{aligned}
\mathbf{G}_{\text {field }} & =\int_{\sigma} \frac{n-\xi_{e}}{c}(\mathbf{E} \times \mathbf{B}) d v \\
\mathbf{G}_{\text {mat }} & =\int_{\sigma} \frac{\xi_{e}}{c}(\mathbf{E} \times \mathbf{B}) d v,
\end{aligned}
$$

is uniquely defined by macroscopic material properties $n$ and $\xi_{e}$.

Since the 1960s, the resolution of the AbrahamMinkowski controversy has been that the total momentum can be arbitrarily separated into field and material components. What has been proved here is that, if the total momentum can be separated into a component for the field and a component for the motion of matter driven by a Lorentz force, then there is a specific form for each of these components in terms of macroscopic material property constants.

\section{MICROSCOPIC TREATMENT OF MATERIAL MOMENTUM}

The total momentum of a closed system with complete equations of motion is unique by virtue of its conservation. It has been argued that the decomposition of the total momentum into field and material is arbitrary [3 -5, 8 13, 18 20]. We now know, as a result of the previous section, that if such a decomposition into a component for the field and a component for the motion of matter driven by a Lorentz force exists then it can be uniquely formulated in terms of macroscopic material property constants. We simply need to determine what those property constants are.

Gordon [9] began with the Lorentz force on a single electric dipole with dipole moment $\mathbf{p}=\alpha \mathbf{e}$

$$
\mathbf{f}_{\text {dipole }}=(\mathbf{p} \cdot \nabla) \mathbf{e}+\frac{d \mathbf{p}}{d(c t)} \times \mathbf{b} .
$$

Here, $\mathbf{e}$ is the microscopic electric field, $\mathbf{b}$ is the microscopic magnetic field, and $\alpha$ is the linear polarizability. Assuming that the distance between dipoles in the material is much smaller than the wavelength, then the theory can be considerably simplified by applying an averaging scheme to a volume containing many atoms per cubic 
radiation wavelength. Gordon [9] describes an averaging process that he uses to derive a macroscopic Lorentz force density

$$
\mathbf{f}_{L}=N\left\langle\mathbf{f}_{\text {dipole }}\right\rangle=\frac{N \alpha}{2}\left(\mathbf{E} \times \frac{\partial \mathbf{B}}{\partial(c t)}+\frac{\partial \mathbf{E}}{\partial(c t)} \times \mathbf{B}\right),
$$

where $N$ is the number density, $\mathbf{E}=\langle\mathbf{e}\rangle$ is the macroscopic electric field, and $\mathbf{B}=\langle\mathbf{b}\rangle$ is the macroscopic magnetic field. Gordon [9] employs the low-density approximation

$$
n=\sqrt{1+N \alpha} \approx 1+N \alpha / 2
$$

such that

$$
\mathbf{f}_{L}=N\left\langle\mathbf{f}_{\text {dipole }}\right\rangle \approx(n-1)\left(\mathbf{E} \times \frac{\partial \mathbf{B}}{\partial(c t)}+\frac{\partial \mathbf{E}}{\partial(c t)} \times \mathbf{B}\right) .
$$

Other authors have derived variations of this basic relation. Peierls [10] used the exact relation

$$
n^{2}=1+N \alpha
$$

to obtain

$$
\mathbf{f}_{L}=N\left\langle\mathbf{f}_{\text {dipole }}\right\rangle=\frac{n^{2}-1}{2}\left(\mathbf{E} \times \frac{\partial \mathbf{B}}{\partial(c t)}+\frac{\partial \mathbf{E}}{\partial(c t)} \times \mathbf{B}\right) .
$$

Saldanha 7] and Bradshaw, et al. 21] find the macroscopic Lorentz force

$$
\mathbf{f}_{L}=\left(n^{2}-1\right)\left(\nabla\left(\frac{1}{2} E^{2}\right)+\mathbf{E} \times \frac{\partial \mathbf{B}}{\partial(c t)}+\frac{\partial \mathbf{E}}{\partial(c t)} \times \mathbf{B}\right) .
$$

If the Lorentz force, Eq. (21), is separated into components,

$$
\begin{gathered}
\mathbf{f}_{L 1}=\left(n^{2}-1\right) \nabla\left(\frac{1}{2} E^{2}\right) \\
\mathbf{f}_{L 2}=\left(n^{2}-1\right)\left(\mathbf{E} \times \frac{\partial \mathbf{B}}{\partial(c t)}+\frac{\partial \mathbf{E}}{\partial(c t)} \times \mathbf{B}\right),
\end{gathered}
$$

then $\mathbf{f}_{L 2}$ is in the form of the Abraham force and differs from the Peierls result for the Lorentz force by a factor of 2 .

We now have three different formulas for the macroscopic Lorentz force, Eqs. (18), (20), and (23), that have been derived from the microscopic Lorentz force on a dipole. Selecting one of these average Lorentz force densities to equate with the macroscopic force density $\mathbf{f}_{\text {mat }}$, Eq. (12), will result in a value for the material parameter $\xi_{e}$ and formulas for the field and material components of momentum in a dielectric, Eqs. (13) and (14). Let us consider the consequences of using each of these formulas, in turn. Comparing the Gordon form of the macroscopic force density, Eq. (18), with Eq. (12) we obtain $\xi_{e}=n-1$. Substituting this result into Eqs. (13) and (14) yields

$$
\mathbf{G}_{\text {field }}=\int_{\sigma} \frac{1}{c}(\mathbf{E} \times \mathbf{B}) d v
$$

$$
\mathbf{G}_{m a t}=\int_{\sigma} \frac{n-1}{c}(\mathbf{E} \times \mathbf{B}) d v, .
$$

The Gordon form of the force, Eq. (18), provides us with the sensible result that the field momentum is the Abraham momentum. But the Gordon form is an approximation. Using Peierls's exact result, Eq. (20), in Eq. (12), we obtain $\xi_{e}=\left(n^{2}-1\right) / 2$. As before, we substitute $\xi_{e}$ into Eqs. (13) and (14) and find that

$$
\begin{gathered}
\mathbf{G}_{f i e l d}=\int_{\sigma} \frac{1+2 n-n^{2}}{2 c}(\mathbf{E} \times \mathbf{B}) d v \\
\mathbf{G}_{\text {mat }}=\int_{\sigma} \frac{n^{2}-1}{2 c}(\mathbf{E} \times \mathbf{B}) d v .
\end{gathered}
$$

Then the Peierls result, Eq. (20), leads to a field momentum, Eq. (26), that is negative for values of the refractive index $n>1+\sqrt{2}$. Further, there is a factor of two discrepancy in the derivations of Gordon [9] and Peierls [10] compared to the later work [7, 21]. Applying the same procedure to the final form of the Lorentz force, Eq. (23), results in

$$
\begin{gathered}
\mathbf{G}_{\text {field }}=\int_{\sigma} \frac{1+n-n^{2}}{c}(\mathbf{E} \times \mathbf{B}) d v \\
\mathbf{G}_{\text {mat }}=\int_{\sigma} \frac{n^{2}-1}{c}(\mathbf{E} \times \mathbf{B}) d v
\end{gathered}
$$

Then, the Lorentz force given by Eq. (23) presents us again with the problem of a negative field momentum for some values of $n$, namely $n>(1+\sqrt{5}) / 2$. Therefore, none of the versions of the Lorentz force that are reprised here from the scientific literature are particularly appealing. Clearly, this procedure to derive the macroscopic Lorentz force is not sufficiently rigorous to obtain a result that is consistent with the total momentum. In the next section, we take a more detailed look at the microscopic Lorentz force.

\section{LAGRANGIAN DYNAMICS OF THE DIELECTRIC}

The macroscopic force density, or Lorentz force, is only significant where the amplitudes of fields are changing in time. The effect of the macroscopic Lorentz force, Eq. (16), has been interpreted, in the context of a typical pulse shape [9]. There is an acceleration of the physical atoms/dipoles on the front side of the pulse where the electromagnetic field is increasing in strength [9]. Then the atoms in the middle portion of the pulse travel at a generally constant velocity until they are decelerated due to the decreasing field strength on the trailing side of the pulse. No momentum is left in the atoms after the pulse has passed. The process has been described by saying that the material momentum travels with the 
pulse [9]. In this section, we derive the dynamics of the atoms/dipoles from the Lagrangian.

The standard Lagrangian for a charged particle with mass $m$ and charge $q$ located at a point $\mathbf{x}$ interacting with the electromagnetic field is given by [27]

$$
\begin{gathered}
L=\frac{1}{2} m \dot{\mathbf{x}}^{2}+\frac{q}{c}(\dot{\mathbf{x}} \cdot \mathbf{A}(\mathbf{x})-\phi(\mathbf{x})) \\
+\int_{\sigma} \frac{1}{2}\left(\left(\frac{1}{c} \frac{\partial \mathbf{A}(\mathbf{x})}{\partial t}+\nabla \phi(\mathbf{x})\right)^{2}-(\nabla \times \mathbf{A}(\mathbf{x}))^{2}\right) d v
\end{gathered}
$$

Here, $\mathbf{A}$ is the vector potential, $\phi$ is the scalar potential and overdots denote partial differentiation with respect to time. We take Eq. (30) as our given starting point.

The derivation and properties of this equation, including the gauge invariance properties, are discussed in Sec. II.B of Ref. [27]. We can construct a Lagrangian for a dipole interacting with the field from a positive charge of mass $m_{+}$located at $\mathbf{x}_{+}$and a negative charge with mass $m_{-}$located at $\mathbf{x}_{-}$and connected by a restoring constant $\kappa$

$$
\begin{gathered}
L=-\frac{1}{2} \kappa\left(\mathbf{x}_{+}-\mathbf{x}_{-}\right)^{2}+\frac{1}{2} m_{+} \dot{\mathbf{x}}_{+}^{2}+\frac{1}{2} m_{-} \dot{\mathbf{x}}_{-}^{2} \\
+\frac{q}{c}\left(\dot{\mathbf{x}}_{+} \cdot \mathbf{A}\left(\mathbf{x}_{+}\right)-\phi\left(\mathbf{x}_{+}\right)-\dot{\mathbf{x}}_{-} \cdot \mathbf{A}\left(\mathbf{x}_{-}\right)+\phi\left(\mathbf{x}_{-}\right)\right) \\
+\int_{\sigma} \frac{1}{2}\left[\left(\frac{1}{c} \frac{\partial \mathbf{A}(\mathbf{x})}{\partial t}+\nabla \phi(\mathbf{x})\right)^{2}-(\nabla \times \mathbf{A}(\mathbf{x}))\right] d v .
\end{gathered}
$$

Transforming to a center-of-mass coordinate system, the Lagrangian is

$$
\begin{gathered}
L=\left[-\frac{1}{2} \kappa \mathbf{r}^{2}+\frac{1}{2} M \dot{\mathbf{R}}^{2}+\frac{1}{2} \mu \dot{\mathbf{r}}^{2}+\frac{q}{c} \dot{\mathbf{r}} \cdot \mathbf{A}(\mathbf{R})\right] \\
+\int_{\sigma} \frac{1}{2}\left[\left(\frac{1}{c} \frac{\partial \mathbf{A}(\mathbf{x})}{\partial t}+\nabla \phi(\mathbf{x})\right)^{2}-(\nabla \times \mathbf{A}(\mathbf{x}))\right] d v .
\end{gathered}
$$

where $M=m_{+}+m_{-}$is the total mass, $\mu=m_{+} m_{-} / M$ is the reduced mass, $\mathbf{r}=\mathbf{x}_{+}-\mathbf{x}_{-}$is the relative coordinate, $\mathbf{R}=\left(m_{+} \mathbf{x}_{+}+m_{-} \mathbf{x}_{-}\right) / M$ is the center-of-mass coordinate. In writing Eq. (32), we have applied the usual dipole approximation in which the electric field does not change much over the distance between charges in the dipole [28], such that

$$
\begin{aligned}
& \mathbf{A}\left(\mathbf{x}_{+}\right) \cong \mathbf{A}\left(\mathbf{x}_{-}\right) \cong \mathbf{A}(\mathbf{R}) \\
& \phi\left(\mathbf{x}_{+}\right) \cong \phi\left(\mathbf{x}_{-}\right) \cong \phi(\mathbf{R})
\end{aligned}
$$

For each dipole in the material, the Lagrange equations of motion are

$$
\frac{d}{d t}\left(\frac{\partial L}{\partial \dot{\mathbf{R}}_{i}}\right)-\frac{\partial L}{\partial \mathbf{R}_{i}}=0
$$

$$
\frac{d}{d t}\left(\frac{\partial L}{\partial \dot{\mathbf{r}}_{i}}\right)-\frac{\partial L}{\partial \mathbf{r}_{i}}=0 .
$$

We will make use of the chain rule

$$
\frac{d}{d t}=\frac{\partial}{\partial t}+\sum \dot{\mathbf{R}}_{i} \frac{\partial}{\partial \mathbf{R}_{i}}+\sum \dot{\mathbf{r}}_{i} \frac{\partial}{\partial \mathbf{r}_{i}} .
$$

Applying Eq. (35) to the Lagrangian, Eq. (32), we obtain a microscopic force equation

$$
\mathbf{f}_{i}=M \ddot{\mathbf{R}}_{i}=\frac{q}{c} \frac{\partial}{\partial \mathbf{R}_{i}}(\dot{\mathbf{r}} \cdot \mathbf{A}(\mathbf{R}))
$$

for each component. Combining the three vector components results in

$$
\mathbf{f}=M \ddot{\mathbf{R}}=\frac{q}{c} \nabla_{R}(\dot{\mathbf{r}} \cdot \mathbf{A}(\mathbf{R}))
$$

where

$$
\nabla_{R}=\left(\frac{\partial}{\partial R_{x}}, \frac{\partial}{\partial R_{y}}, \frac{\partial}{\partial R_{z}}\right)
$$

Applying the vector identity

$$
\nabla(\mathbf{A} \cdot \mathbf{B})=(\mathbf{B} \cdot \nabla) \mathbf{A}+(\mathbf{A} \cdot \nabla) \mathbf{B}+\mathbf{B} \times(\nabla \times \mathbf{A})+\mathbf{A} \times(\nabla \times \mathbf{B})
$$

in the plane-wave limit, we obtain the microscopic force equation

$$
\mathbf{f}_{\text {dipole }}=M \ddot{\mathbf{R}}=\frac{q}{c} \dot{\mathbf{r}} \times\left(\nabla_{R} \times \mathbf{A}(\mathbf{R})\right)
$$

that is the Lorentz force on a single dipole, that is exerted at the center of mass. In the plane-wave limit, the Lorentz force is in the direction of propagation, or the opposite direction, such that $\nabla_{R}=\nabla$ without loss of generality. With definitions for the magnetic field $\mathbf{b}=\nabla \times \mathbf{A}$, and the polarizability $\mathbf{p}=q \mathbf{r}$, we have the Lorentz force on a dipole

$$
\mathbf{f}_{\text {dipole }}=M \ddot{\mathbf{R}}=\frac{1}{c} \dot{\mathbf{p}} \times \mathbf{b}
$$

The Lorentz force on a dipole that is given by Eq. (15) contains an extra term $(\mathbf{p} \cdot \nabla) \mathbf{e}$ that does not appear in the Lorentz force, Eq. (43). This term went out in the dipole approximation, which is fortunate because the dipole force should not have a transverse component in the plane-wave limit.

In the Gordon [9] model, the atoms are free particles that are accelerated by the Lorentz force at the leading edge of the field and travel at constant velocity until decelerated by the Lorentz force at the trailing edge of the pulse. Then, the momentum of the atoms travels with the field and contributes to the total momentum. However, that model assumes a low-density limit in which there is no scattering of the field and assumes that the atoms move without being restrained by association with a lattice and without colliding with any other particles 
in the material, i.e. a vacuum. Instead, there is substantial impediment to the motion of atoms in a dielectric that, after all, is a material that can be modeled as a continuous medium. Then the canonical momentum

$$
M \dot{\mathbf{R}}=\left(\frac{\partial L}{\partial \dot{\mathbf{R}}_{1}}, \frac{\partial L}{\partial \dot{\mathbf{R}}_{2}}, \frac{\partial L}{\partial \dot{\mathbf{R}}_{3}}\right)
$$

makes no appreciable contribution to the total momentum.

The other Lagrange equation of motion

$$
\mu \ddot{\mathbf{r}}+\kappa \mathbf{r}=-\frac{q}{c} \dot{\mathbf{A}}-\frac{q}{c}\left(\dot{\mathbf{R}} \cdot \nabla_{r}\right) \mathbf{A}
$$

that is derived by applying Eq. (36) to the Lagrangian, Eq. (30), and collecting the components is more interesting because it is evocative of the other microscopic model of a dielectric as stationary simple harmonic oscillators driven by the electric field [24]. Setting $\dot{\mathbf{R}}=0$ in Eq. (45) we recover the usual and familiar model of a dielectric as a collection of stationary simple harmonic oscillators that are driven by the electric field [24]. Defining a frequency $\omega_{0}=\sqrt{\kappa / \mu}$, the solution of

$$
\ddot{\mathbf{p}}+\omega_{0}^{2} \mathbf{p}=-\frac{q^{2}}{\mu c} \dot{\mathbf{A}}=\frac{q^{2}}{\mu} \mathbf{e}
$$

for a time harmonic field $\mathbf{e}=\left(\tilde{\mathbf{e}} e^{-i\left(\omega_{p} t-k z\right)}+\right.$ c.c. $) / 2$ is

$$
\tilde{\mathbf{p}}=\frac{q^{2} / \mu}{\omega_{0}^{2}-\omega_{p}^{2}} \tilde{\mathbf{e}}
$$

Assuming the same relation holds for macroscopic fields, we have a macroscopic polarization field

$$
\tilde{\mathbf{P}}=\frac{N q^{2} / \mu}{\omega_{0}^{2}-\omega_{p}^{2}} \tilde{\mathbf{E}}=\chi \tilde{\mathbf{E}}
$$

given a number density of dipoles $N$. The fields, electric, magnetic, and polarization, travel together as a pulse of electromagnetic radiation.

It is well known that the polarization field contributes to the electromagnetic energy

$$
U_{e}=\int_{\sigma} \frac{1}{2}\left((\mathbf{E}+\mathbf{P}) \mathbf{E}+\mathbf{B}^{2}\right) d v,
$$

as well as the electric and magnetic fields. In terms of time-harmonic fields, the electromagnetic energy density is

$$
\rho_{e}=n^{2} \tilde{E}^{2} .
$$

Although a material may possess many forms of energy, we intend total energy to include only those forms of energy that could impact the dynamics or electrodynamics of the model system. If the material is initially stationary in the laboratory frame of reference, then it remains stationary with negligible kinetic energy due to the gradient-index antireflection coating. Then the electromagnetic energy is the total energy for a stationary linear dielectric.

Momentum is associated with the movement of energy. The electromagnetic momentum will contain a contribution from the movement of the polarization field, as well as contributions from the movement of the electric and magnetic fields. Writing the total momentum density, Eq. (3), in terms of time-harmonic fields, we have

$$
\mathbf{g}_{\text {total }}=\frac{n^{2} \tilde{E}^{2}}{c^{2}} c \hat{\mathbf{e}}_{\mathbf{k}}=\frac{\rho_{e}}{c^{2}} c \hat{\mathbf{e}}_{\mathbf{k}}
$$

where $\hat{\mathbf{e}}_{\mathbf{k}}$ is a unit vector in the direction of propagation. Then, in a stationary dielectric medium, the equality of the total momentum density and the electromagnetic energy density means that the total momentum density, Eq. (3), is equal to the electromagnetic momentum density, Eq. (13), that is, $\mathbf{G}_{\text {field }}=\mathbf{G}_{\text {total }}$. Then we have $\xi_{e}=0$ and substituting this result into Eq. (14), we find $\mathbf{G}_{\text {mat }}=0$. There is no appreciable matter component of either energy or momentum for a simple anti-reflection coated linear dielectric medium that is at rest in the laboratory frame of reference.

The situation changes somewhat in the absence of a gradient-index antireflection coating. Momentum balance requires, at the cost of some amount of rigor in the derivation, that we impute a momentum to the material that is twice the momentum of the reflected field imparted by the surface force of reflection. But, that is the only significant material momentum, there is no momentum of the Gordon type due to motion of the atoms inside the dielectric.

\section{CONCLUSIONS}

The total momentum is uniquely conserved in a thermodynamically closed system with complete equations of motion. Once the total momentum is known, with certainty, from conservation principles, then the momentum relations can be derived. Using a microscopic model of the dielectric medium, we showed that the propagating polarization field, not the movement of matter, is a component of the momentum of a system consisting of a stationary dielectric illuminated by a pulse of quasimonochromatic radiation through a gradient-index antireflection coating. 
[3] I. Brevik, Phys. Rep. 52, 133 (1979).

[4] P. Penfield, Jr. and H. A. Haus, Electrodynamics of Moving Media (MIT Press, 1967).

[5] C. Møller, The Theory of Relativity, (Clarendon, 1962).

[6] M. Kranyš, Int. J. Engng. Sci. 20, 1193 (1982).

[7] P. L. Saldanha, Opt. Commun. 284, 2653 (2011).

[8] R. N. C. Pfeifer, T. A. Nieminen, N. R. Heckenberg, and H. Rubinsztein-Dunlop, Rev. Mod. Phys. 79, 1197 (2007).

[9] J. P. Gordon, Phys. Rev. A 8, 14 (1973).

[10] R. Peierls, Proc. R. Soc. Lond. A 347, 475 (1976).

[11] S. M. Barnett and R. Loudon, Phil. Trans. R. Soc. A 368, 927 (2010).

[12] P. W. Milonni and R. W. Boyd, Adv. Opt. Photon. 2, 519 (2010).

[13] B. A. Kemp, J. Appl. Phys. 109, 111101 (2011).

[14] D. J. Griffiths, Am. J. Phys. 80, 7 (2012).

[15] W. She, J Yu, R. Feng, Phys. Rev. Lett. 101, 243601 (2008).

[16] A. Ashkin and J. M. Dziedzici, Phys. Rev. Lett. 30, 139 (1973).

[17] T. Ramos, G. F. Rubilar, and Y. N. Obukhov, Phys.
Lett. A 375, 1703 (2011).

[18] S. M. Barnett, Phys. Rev. Lett. 104, 070401 (2010).

[19] M. Mansuripur, Opt. Expr. 12, 5375 (2004).

[20] B. A. Kemp, Proc. SPIE 8458, Optical Trapping and Optical Micromanipulation IX, 845803 (2012).

[21] D. H. Bradshaw, Z. Shi, R. W. Boyd, P. W. Milonni, Opt. Commun. 283, 650 (2010).

[22] M. E. Crenshaw and T. B. Bahder, Opt. Commun. 284, 2460 (2011).

[23] M. E. Crenshaw, Proc. SPIE 8458, Optical Trapping and Optical Micromanipulation IX, 845804 (2012).

[24] M. V. Klein, Optics, (Wiley, 1970).

[25] W. Rindler, Introduction to Special Relativity, (Clarendon, 1982).

[26] Z. Mikura, Phys. Rev. A 13, 2265 (1976).

[27] C. Choen-Tannoudji, J. Dupont-Roc, and G. Grynberg, Photons and Atoms: Introduction to Quantum Electrodynamics (Wiley, 1989).

[28] W. P. Schleich, Quantum Optics in Phase Space, (Wiley$\mathrm{VCH}, 2001)$ 